\title{
Corela
}

Cognition, représentation, langage

HS-8 | 2010

L'interpellation

\section{Les questions toniques : une forme d'interrogation interpellative}

Carole Lailler

\section{OpenEdition}

Journals

Édition électronique

URL : http://journals.openedition.org/corela/1688

DOI : $10.4000 /$ corela. 1688

ISSN : 1638-573X

Éditeur

Cercle linguistique du Centre et de l'Ouest - CerLICO

Référence électronique

Carole Lailler, «Les questions toniques : une forme d'interrogation interpellative », Corela [En ligne], HS-8 | 2010, mis en ligne le 23 novembre 2010, consulté le 30 avril 2019. URL : http:// journals.openedition.org/corela/1688; DOI : 10.4000/corela.1688

Ce document a été généré automatiquement le 30 avril 2019.

\section{(c) (i) (2)(2)}

Corela - cognition, représentation, langage est mis à disposition selon les termes de la licence Creative Commons Attribution - Pas d'Utilisation Commerciale - Partage dans les Mêmes Conditions 4.0 International. 


\title{
Les questions toniques : une forme d'interrogation interpellative
}

\author{
Carole Lailler
}

\section{Introduction}

1 La Grammaire Interactive (à présent notée GI) constitue un cadre théorique nouveau qui a pour but de cartographier les variations morphosyntaxiques de l'intentionnalité dans le cadre de l'interrogation en français contemporain. Cette grammaire, qui se veut avant tout interprétative et dynamique, présuppose qu'un lien de dépendance direct existe entre la morphosyntaxe de l'interrogation et une "réponse escomptée » préexistante dans l'esprit du locuteur. Ce dernier formulerait sa question en fonction de cette « réponse escomptée » attendue et de l'orientation qu'il souhaite donner à son message. En analysant les nombreuses variations morphosyntaxiques autour d'un même objet interrogé (le lieu, le temps, la quantification, les causes, etc.), on serait alors en mesure d'évaluer ce qui est réellement questionné par le locuteur : l'objet même de la question, sa réalité, voire sa légitimité et celle de l'interlocuteur, destinataire de la question. Cette théorie, qui s'intéresse moins à la normativité d'une forme qu'à la description de l'utilisation réelle de la langue, se fonde sur la morphosyntaxe des énoncés pour aborder le contenu intentionnel du langage en interaction, à la différence des approches essentiellement planificatrices fondées sur les théories des Actes de Langage [Searle, 1996].

2 De récents travaux sur la proéminence [Avanzi, Goldman, Lacheret-Dujour, Simon et Auchlin, 2007] ont confirmé que les questions toniques se caractérisent par un accent porté et/ou perçu sur la syllabe finale qui correspond au mot interrogatif: Tu vas où ? Tu fais quoi? Tu t'appelles comment? Tu viens quand? Cet accent revient à interpeller l'interlocuteur, à attirer son attention, par opposition notamment aux formulations concurrentes: Où vas-tu? Que fais-tu? Où est-ce que tu vas? Je voudrais savoir comment tu t'appelles?, etc. 
3 Les questions toniques, bien que rares dans les corpora, constituent une véritable interpellation de l'interlocuteur qui se voit ainsi lui-même mis en cause. On exige de lui qu'il se justifie quant à son action, son déplacement, son propos, etc. Ces questions sont autant «d'attaques » sur les "faces» de l'interlocuteur: mettant à mal la "politesse » requise dans un échange oral spontané [Kerbrat-Orecchioni, 2005], la formulation tonique d'une interrogation autorise le locuteur à montrer son impatience vis-à-vis de l'attitude, des requêtes ou, plus généralement, des propos tenus par l'interlocuteur.

4 La GI, afin d'apprécier au plus près les variations de l'intentionnalité d'un locuteur, utilise des modèles permettant de cartographier la totalité du paradigme de l'interrogation. Ainsi, ces modèles se composent de deux axes; le premier, appelé " axe ontologique " traite de l'objet de la question à travers les représentations de la connaissance au sujet d'un monde : s'agit-il, par exemple, d'une question locative, temporelle ou quantificatrice et comment se réalise-t-elle (localisation, spatialisation, temps, durée, espace-temps défini, mesure, etc.) ? Cet axe est infini mais cependant balisable car les variations possibles dépendent de l'objet de la question. Ainsi, une question locative n'autorise pas de formulation numérale tandis que les questions temporelles et quantificatrices l'admettent de fait (Combien d'euros coûte cette voiture? et A quelle heure part ce train ?).

5 Le second axe, appelé axe "interrogatif», répertorie les différentes précautions et politesses mises en œuvre par un locuteur en classifiant les réalisations morphosyntaxiques auxquelles on aboutit. Il s'agit là encore d'un axe infini mais cependant balisable selon les utilisations langagières rencontrées. Toutefois, une opposition majeure s'impose au sein de cet axe : celle existant entre interrogation tonique (Tu fais quoi ?) et interrogation périphrastique (Je voudrais savoir ce que tu fais?).

\section{Grammaire Interactive et interpellation}

\subsection{Les modèles théoriques de la GI}

6 Chaque modèle théorique de la GI correspond à un objet du monde mis en question. Ont été ainsi développés jusqu'à maintenant les modèles théoriques des questions quantificatrices, temporelles et locatives. Un dernier modèle concernant les questions nominales est en cours d'expérimentation. Nous y reviendrons plus avant dans notre article.

7 Si l'on s'intéresse plus particulièrement, par exemple, au modèle théorique des questions locatives, on voit que ce dernier comporte cinq possibilités morphosyntaxiques de poser une question concernant le lieu ou, du moins, une localisation. Cet axe morphosyntaxique reste identique quel que soit l'objet mis en question. On peut simplement souligner que le premier type, qualifié de "prototypique», est rarement utilisé par les locuteurs, vraisemblablement compte-tenu de son caractère trop figé et normé. Il s'agit avant tout d'une formulation "grammaticale » telle qu'on les trouve dans les manuels scolaires. La dernière forme, dite forme assertive, est, quant à elle, à la limite même de l'interrogation. En effet, seule l'intensité, l'accentuation et le contexte permettent de conclure à une interrogation. Le locuteur, en ayant recours à ce type de formulation, cherche à trouver confirmation auprès de son interlocuteur. Le type "renforcé ", qui utilise la locution " est-ce que ", représente la véritable interrogation sur l'objet du monde. Avec ce type, le locuteur met véritablement en question le lieu et non sa réalité ou bien l'interlocuteur 
lui-même. Nous sommes confrontés à un type qui marque morphosyntaxiquement la volonté de connaître le lieu ou la localisation et non autre chose.

Outre ces trois formes morphosyntaxiques, un locuteur peut se servir de deux autres formes. Ces deux dernières, la forme tonique et la forme périphrastique, possèdent un point commun qui nous permettra d'ailleurs de les opposer. Elles visent toutes deux, à des degrés différents, voire contradictoires, à mettre en cause l'interlocuteur.

\begin{tabular}{|c|c|c|c|c|}
\hline & $\begin{array}{c}\text { la } \\
\text { adverbiale }\end{array}$ & $\begin{array}{c}\text { Ib } \\
\text { déterminative }\end{array}$ & $\begin{array}{c}\text { Ic } \\
\text { confirmative }\end{array}$ & Objet visé \\
\hline $\begin{array}{c}\text { 2a } \\
\text { prototypique } \\
\mathrm{Mi}+\mathrm{vbe}+\mathrm{X} \text { ? }\end{array}$ & Où part Jean? & $\begin{array}{l}\text { A quel endroit } \\
\text { part Jean ? }\end{array}$ & $\begin{array}{l}\text { Jean part-il à } \\
\text { Lyon? }\end{array}$ & $\begin{array}{l}\text { Repérage } \\
\text { locatif }\end{array}$ \\
\hline \begin{tabular}{l}
\multicolumn{2}{c}{$\mathrm{b}$} \\
tonique \\
$\mathrm{X}+\mathrm{vbc}+\mathrm{Mi}$ \\
tonique?
\end{tabular} & Jean part où? & $\begin{array}{c}\text { Jean part } \\
\text { à quel endroit ? }\end{array}$ & $\begin{array}{l}\text { C'est bien à Lyon } \\
\text { que part Jean? }\end{array}$ & $\begin{array}{l}\text { Réalité du lieu } \\
\text { Alternative } \\
\text { possible }\end{array}$ \\
\hline 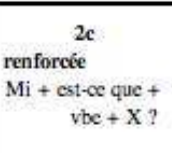 & $\begin{array}{l}\text { Où est-oe que } \\
\text { part Jean? }\end{array}$ & $\begin{array}{l}\text { A quel endroit } \\
\text { est-o que part } \\
\text { Jean? }\end{array}$ & $\begin{array}{l}\text { Est-oe que } \\
\text { Jean part } \\
\text { (bien) a Lyon ? }\end{array}$ & $\begin{array}{c}\text { LIEU } \\
\text { Localisation } \\
\text { Lieu ou } \\
\text { spatialisatI } \\
\text { on }\end{array}$ \\
\hline $\begin{array}{c}\mathbf{2 d} \\
\text { périphrastique. } \\
\text { Je vds savoir }+ \\
\mathrm{Mi}+\mathrm{vbc}+\mathrm{X} \text { ? }\end{array}$ & $\begin{array}{l}\text { Je voudrais savoir } \\
\text { où part Jean? }\end{array}$ & $\begin{array}{l}\text { Je voudrais savoir } \\
\text { à quel endroit } \\
\text { Jean part ? }\end{array}$ & $\begin{array}{l}\text { Je voudrais savoir } \\
\text { si Jean part } \\
\text { à Lyon ou non? }\end{array}$ & $\begin{array}{l}\text { Connaissance } \\
\text { de la réponse }\end{array}$ \\
\hline $\begin{array}{c}2 \mathrm{e} \\
\text { assertive } \\
X+v b e+R\end{array}$ & $\begin{array}{l}\text { Jean part (bien) } \\
\text { à Lyon? }\end{array}$ & $\begin{array}{c}\text { Jean part } \\
\text { à la campagne. (?) }\end{array}$ & $\begin{array}{l}\text { C'est bien à Lyon } \\
\text { que part Jean? }\end{array}$ & $\begin{array}{l}\text { Confirmation } \\
\text { ou } \\
\text { infirmation }\end{array}$ \\
\hline Réponse & $\begin{array}{c}\text { "à Lyon } x / \text { / il } \\
\text { ne part } \\
\text { pas * } \\
\text { neutralité }\end{array}$ & $\begin{array}{r}\text { * loin *- " pas } \\
\text { loin * / } \\
\text { \& aucune } \\
\text { idce * } \\
\text { estimation }\end{array}$ & $\begin{array}{c}\text { « oui } \approx / \text { \& pas du } \\
\text { tout } \% \\
\text { confirmation/ } \\
\text { infirmation }\end{array}$ & $\begin{array}{l}\text { Idem pour les } \\
\text { préposition } \\
5 \\
\text { * d'ou ....* * }\end{array}$ \\
\hline
\end{tabular}

Figure 1

Exemple d'un modèle théorique de la Grammaire Interactive concernant les questions locatives

\subsection{Une opposition périphrastique/tonique}

En examinant attentivement les variations morphosyntaxiques, on remarque le lien existant entre les formulations périphrastiques et toniques. L'interrogation périphrastique Je voudrais savoir où se trouve la Tour Eiffel? permet de ménager l'interlocuteur. Quelle que soit la tournure syntaxique choisie autour du groupe verbal (Je voudrais savoir entre en concurrence avec Il faudrait absolument que vous me disiez ou Auriezvous l'obligeance de me dire, etc.), il s'agit d'une formulation qui dédouane par avance l'interlocuteur d'un défaut de réponse. Il a le droit de ne pas savoir, de ne pas pouvoir dire, voire de ne pas vouloir répondre. Il n'y a donc pas d'interpellation du destinataire du message ; l'émetteur tente d'appréhender l'objet visé - ici, savoir localiser la Tour Eiffel - mais il le fait en rassurant son interlocuteur, c'est-à-dire en ménageant sa face négative. Il n'est nullement question de mettre en défaut cet interlocuteur ; il s'agit en tout état de cause de se ménager une collaboration.

L'interrogation tonique La Tour Eiffel, c'est où?, à l'inverse, est une interrogation interpellative: elle prend à partie l'interlocuteur et peut constituer une forme 
d'agression. Certes, l'objectif du locuteur n'est pas nécessairement d'agresser physiquement le destinataire, mais il l'intimide, en affichant une éventuelle mise en cause de sa légitimité à ne pas répondre. Le locuteur interpelle son allocutaire en le déstabilisant et en attaquant sa Face positive. Par ce procédé morphosyntaxique, on tente moins d'appréhender l'objet visé que de prendre en défaut l'interlocuteur.

Ces deux types de questions permettent de mettre en lumière le lien extrêmement ténu existant dans une conversation orale spontanée ponctuée de questions entre les deux interactants. Chacun cherche à établir une relation avec son allocutaire tout en maintenant une recherche d'informations effective. Toutefois, dans le cas des interrogations périphrastiques et toniques, il s'agit d'abord et avant tout de se placer par rapport à l'autre et de marquer son territoire, de lui faire sentir son aptitude à approuver ou à s'opposer.

12 Ainsi, dans le cas d'une situation de classe où un des élèves se lève sans la permission de son professeur, ce dernier lui demandera certainement $T u$ vas où ?. Il s'agira pour l'adulte de signifier au trublion que son déplacement est ressenti comme injustifié et surtout illégitime. De même, lorsqu'un employé de la SNCF demande à un client C'est pour aller où ? , il l'interpelle en lui montrant que l'objectif de l'échange est l'annonce de la destination et non toute autre question annexe. Il le met ainsi en défaut, par exemple, de ne pas savoir exactement où il veut aller, de pas l'énoncer clairement ou de tergiverser sur le prix du trajet.

\subsection{L'interpellation}

13 L'interpellation peut se définir selon trois aspects complémentaires. Tout d'abord, il s'agit d'une définition subjective. L'interpellation se trouve dans l'effet produit via une forme morphosyntaxique et traduit l'intentionnalité première du locuteur, éventuellement non consciente. Le sujet est donc impliqué dans la formulation qu'il choisit et cette implication autorise une confrontation avec l'allocutaire. S'agissant d'oral spontané, le retravail de l'énoncé n'est possible qu'en un second temps. L'intentionnalité est véhiculée sans fard.

Ensuite, l'interpellation telle que nous l'entendons se comprend selon une définition interactive. En effet, l'interlocuteur est interpellé et la formulation morphosyntaxique ne concerne pas tant l'objet visé par la question que la mise en cause du destinataire. Enfin, une définition contrastive est ici convoquée puisque les questions toniques ou " questions interpellatives » s'opposent aux questions périphrastiques, les plus "polies». De plus, elles se distinguent notamment d'une véritable question sur l'objet visé, faisant davantage abstraction de l'allocutaire et de forme plus standard de type Où est-ce que se trouve la Tour Eiffel?

15 Par ailleurs, la forme morphosyntaxique interpellative s'oppose à d'autres approches, qui elles aussi prennent en compte l'interlocuteur et tentent de l'impliquer dans la conversation. Il en va ainsi pour l'approche syntaxique, marquée notamment par le type phrasoïde avec les interjections, véritables mots phrases Attention! En outre, les procédés de topicalisation C'est bien la souris que le chat mange? se différencient du simple détachement et s'opposent donc à la forme interpellative en n'impliquant pas l'interlocuteur comme individu mais comme interface de réponse. L'approche sémantique est une autre forme qui s'oppose à la morphosyntaxe. En ayant recours à la prédication seconde Et ce rendez-vous que vous m'aviez promis? voire à la prédication impliquée ou 
incomplète Allô?, le locuteur amorce une conversation en se mettant lui-même au premier plan.En dernier lieu, les différentes formes de dénomination ou appellatifs Mon petit, tu... ainsi que les procédés d'intimation Non, fais pas ça! offrent la possibilité d'une alternative pragmatique pour apostropher autrui.

La forme interpellative a, en outre, le mérite d'intégrer intrinsèquement la dimension prosodique. De fait, une formulation tonique appartient à l'oralité mais cette tonicité n'est pas uniquement prosodique ou ponctuée, les marques de ponctuation étant par ailleurs assez peu discriminantes. L'opposition tonique/atone permet de marquer syntaxiquement la tonicité. Cette opposition est symboliquement représentée par l'opposition QUE/QUOI dans les questions Que fais-tu? [question prototypique véhiculant le maximum de neutralité] et $T u$ fais quoi ? [question tonique marquant la désapprobation du locuteur sur l'occupation (ou l'inoccupation) de son interlocuteur]. Bien sûr, on peut obtenir un "effet interpellatif ", perceptible même à l'écrit, grâce à un connecteur comme donc Quand viens-tu donc?, aux procédés de topicalisation C'est quoi c'que tu manges? ou bien au morphème -ti C'est-ti vrai tout ça? mais d'une part il s'agit toujours de formulation à tonicité latente, et d'autre part cet effet doit être distingué des questions de forme tonique à visée essentiellement interpellative.

17 La Grammaire Interactive permet de construire et d'isoler des paradigmes selon les types de questions. Par conséquent, il existe pour chaque type d'interrogation, sa variation tonique interpellative et c'est précisément cette catégorie de questions que nous nous proposons d'examiner dans des corpora.

\section{Le corpus RITEL et sa " géographie »}

Les variations morphosyntaxiques sont autant de manifestations identifiables de l'intentionnalité du locuteur. Avec ce cadre théorique, il ne s'agit pas d'appréhender un «objet question" mais de prendre en considération un processus dynamique. La GI établit de fait un contact direct avec la forme des énoncés. On pourra évaluer une utilisation réelle de la langue à travers ces énoncés avec l'examen minutieux des corpora qui permettront l'enrichissement puis la validation des modèles. Ce lien représente un avantage certain pour le DOHM ; ces énoncés étant facilement repérables et identifiables morphosyntaxiquement. Le corpus RITEL est justement un corpus de DOHM pour lequel la GI tente une cartographie.

\subsection{Un corpus de DOHM}

En mesurant l'intention qui est véhiculée par des énoncés perçus et conçus au fil de leur énonciation, les modèles de la GI sont un outil au service du dialogue oral spontané. RITEL est un corpus construit artificiellement et non pensé pour la GI, issu d'un système de DOHM en Question-Réponse. C'est un objet de travail façonné par les requêtes utilisateurs du LIMSI : 13 personnes ont reçu une liste de 300 modèles de questions ce qui a donné lieu à la production de 582 «dialogues»[Rosset, Galibert, Illouz et Max, 2005]. Ces «dialogues» sont le fruit d'une «pseudo-conversation» entre un locuteur humain soucieux d'être compris et désireux d'obtenir une réponse et un interlocuteur machine en situation d'apprentissage. Ce corpus représente un total de 5362 énoncés presque intégralement en modalité interrogative. Certaines ouvertures de "cohortes" sont en effet réalisées en modalité assertive puisqu'il s'agit de ce qu'il convient d'appeler des pré- 
interrogations du type Je voudrais des renseignements sur la mort de James Dean. On peut certes reprocher à ce corpus d'être biaisé dans la mesure où l'interlocuteur machine ne « répond» pas. En revanche, cet aspect possède un précieux avantage : il n'y a pas de «redirection dialogique» aux questions posées initialement, ce qui permet aux « cohortes » d'apparaître et d'être complètes. En effet, le simple fait de proposer une amorce de réponse ou de demander au locuteur, par exemple, de reformuler sa question, peut influer sur l'intentionnalité première. L'interactant, voyant le dialogue s'amorcer, peut être tenté d'infléchir sa démarche et d'entrer dans une conversation dont les objectifs ne seront pas seulement pragmatiques, à savoir l'efficacité du dialogue mais pourront satisfaire d'autres buts comme la courtoisie, l'échange diplomatique, etc.

Le corpus de DOHM RITEL doit être considéré, on le voit, comme un outil de falsification nécessaire bien que partiel puisque la machine ne répond pas à son interlocuteur humain. Il permet cependant la validation des modèles grammaticaux par les productions ellesmêmes. Il constitue un objet analysable puisque les énoncés ont été réellement construits par un locuteur soucieux de sa formulation. Par ailleurs, un phénomène de "cohortes » autorise une analyse par segments.

\subsection{Les « cohortes»}

Les «cohortes» correspondent à l'apparition d'une séquence itérative d'une même question autour du même propos ou thème. Chaque séquence offre donc la possibilité d'étudier précisément les variations morphosyntaxiques d'une même interrogation et d'en mesurer les fréquences. Ainsi, les questions périphrastiques sont pléthores comptetenu du fait que le locuteur humain essaie d'être le plus clair et le plus mesuré possible tandis que les questions toniques n'interviennent qu'en fin de cohorte, juste avant la rupture. Oubliant que la machine n'est qu'un « locuteur restreint », le questionneur perd patience et l'interpelle pour tenter d'obtenir enfin une réponse.

- en fait je vais m'intéresser plutôt à Toulouse et je voudrais savoir où est Toulouse : question locative adverbiale périphrastique = interrogation polie du locuteur qui dédouane l'interlocuteur/début de cohorte, volonté d'amorce claire et polie

- Où est située la ville de Toulouse : question adverbiale prototypique = question grammaticalement normée qui trahit la volonté d'être compris

- dans quel pays est Toulouse : question déterminative prototypique = variation ontologique la précédente question pour les mêmes effets

- je voudrais connaître la situation géographique de Toulouse : question rhétorique périphrastique = information donnée à l'interlocuteur machine pour circonscrire le domaine

- Toulouse est une ville qui est en Europe mais dans quel pays: question déterminative tonique précédée d'une explicitation avec relative déterminative agacement certain devant l'incompréhension et l'absence de réponse de l'interlocuteur machine

Figure 2

Exemple de «cohorte » au sein du corpus RITEL

\subsection{Les résultats}

Sur les 5362 énoncés présents dans le corpus et analysés par le prisme de la GI, les questions quantificatrices, locatives et temporelles représentent, au total, $13,31 \%$. Il 
s'agit là d'une couverture faible mais qui montre bien que ce type de question, à savoir les questions où le mot interrogatif invite à localiser spatialement, temporellement et quantitativement un actant du verbe, n'est pas prioritairement utilisé par les locuteurs. Ces questions sont précises et émanent d'un interactant soucieux d'obtenir un réponse pour mener à bien une recherche extérieure à la conversation (trouver Jean, la Tour Eiffel, savoir ce que vaut la voiture pour pouvoir demander un prêt, savoir à quelle heure part son train pour demander à ce qu'on vienne le chercher à l'arrivée, etc.) Cette recherche est d'abord pragmatique et vise essentiellement la mise en place des conditions d'une réalisation effective. De ce fait, ces questions sont moins nombreuses, même s'il est clair qu'on peut toujours poser une question locative, quantificatrice ou temporelle dans un but encyclopédique. Une conversation orale dans un cadre spontané laisse généralement la part belle à l'interaction et non à l'expression d'un sujet unique. C'est pourquoi les questions toniques, bien que peu nombreuses interviennent prioritairement et quasi-exclusivement en fin de « cohortes ».

\subsubsection{Taux de couverture des questions toniques}

23 Ce constat révèle, une fois encore, le caractère interpellatif des questions toniques : le locuteur a épuisé toutes les autres formes morphosyntaxiques à sa disposition et décide de prendre à partie son interlocuteur, oubliant quelques instants qu'il ne s'agit que d'une machine. Il la somme de lui répondre et ne peut comprendre son silence devant la clarté de la question posée.

Le tableau comparatif ci-dessous révèle l'omniprésence des questions de type périphrastique. Sur les $13,31 \%$ de couverture offert par le regroupement des questions cherchant à «mesurer » (quantitativement, spatialement et temporellement) les objets du monde, presque $63 \%$ des questions sont posées de façon périphrastique, ce qui s'explique vraisemblablement par une volonté consciente du locuteur de ne pas «agresser » une machine dont l'intelligence n'est qu'artificielle! Les $33 \%$ restant représentent les autres types morphosyntaxiques confondus.

\begin{tabular}{|c|c|}
\hline Types de questions & Résultats \\
\hline QQ toniques & $\mathbf{0 , 6 3 \%}$ \\
\hline QT toniques & $\mathbf{3 , 9 1 \%}$ \\
\hline QL toniques & $\mathbf{1 0 , 4 1 \%}$ \\
\hline Soit $13,31 \%$ & $\mathbf{4 , 4 4 \%}$ \\
\hline Types de questions & Résultats \\
\hline QQ periphr. & $\mathbf{7 9 , 6 1 \%}$ \\
\hline QT periphr. & $\mathbf{6 6 , 6 6 \%}$ \\
\hline QL periphr. & $42,36 \%$ \\
\hline Soit $13,31 \%$ & $\mathbf{6 2 , 8 8 \%}$ \\
\hline
\end{tabular}


Figure 3

Tableau comparatif des résultats obtenus pour les deux types morphosyntaxiques de questions en opposition permet une description, voire une interprétation. Enfin, cette dernière interrogation montre que les questions cherchant à nommer en les catégorisant les objets du monde (question de type nominal) sont plus nombreuses encore que les questions mettant en débat la mesure (avec les mots interrogatifs quand, où, et combien) ou la cause (avec les mots interrogatifs comment et pourquoi) des objets en question. C'est justement ce type de questions nominales, traitées via le concept restreint "d'Entités Nommées", qui constituent l'étape à venir, susceptible d'améliorer sensiblement le taux de couverture de RITEL.

\subsubsection{Les Entités Nommées}

Les questions nominales sont utilisées pour catégoriser le monde en nommant les realia qui le composent; le locuteur cherche à connaître un actant du verbe mis en question. Ainsi, demander à quelqu'un Que manges-tu ?, c'est chercher à connaître le second actant du verbe manger, à savoir préférentiellement un Inanimé « comestible »! La réponse Des haricots verts correspond à une question de type «Comment s'appelle » pour laquelle le concept des Entités Nommées [Fourour, 2004], du moins dans sa définition la moins large, apporte des solutions de classement. Nous entendons par classement en Entités nommées, une catégorisation des noms qui possèdent plus ou moins un référent unique. Nous retiendrons donc pour le classement de ces questions, les réponses en ENPersonne ( Qui fut élu Président de la République en 1981 ? - François Mitterand = ENPers.), en ENLieu (Quel est le monument le plus visité de la capitale? - La Tour Eiffel = ENLieu), et en ENObjet (Que manges-tu $?$ Des frites $=$ ENObjet $)$.

Tout comme pour les types de questions déjà examinés, les questions nominales font l'objet d'un traitement selon les variations morphosyntaxiques. Il existe bel et bien une version tonique pour ce type de question. On peut alors constater qu'il existe toujours la même dualité tonique/atone (quelle vs laquelle), reprise notamment dans l'opposition entre formulation périphrastique Je voudrais savoir quelle est le plus haute montagne du monde? et formulation tonique La plus haute montagne du monde, c'est laquelle? Présentes également en fin de « cohortes », les questions nominales toniques cherchent à mettre en défaut l'interlocuteur en lui prouvant son peu de connaissances. Or, ne pas savoir nommer les réalités qui nous entourent constitue rapidement un manque que le locuteur attribue à une faiblesse pour le moindre, à une insuffisance voire, pour le pire, à une provocation délibérée de l'allocutaire. Ce petit jeu de domination entre les deux 
interactants mène le plus souvent à la rupture de l'échange, au détriment de la réponse elle-même comme on le voit dans l'exemple suivant.

- je voudrais poser une question sur le sport: détermination du contexte, volonté affichée du locuteur de baliser son intervention / pré-interrogative

- je sais qu'un seul pays a participé à tous les Jo d'hiver et j'aimerais savoir lequel: affirmation d'une connaissance, ce qui réduit le champ d'action de l'interlocuteur machine - recherche d'une EN Lieu avec une question nominale périphrastique tonique $=$ volonté $d u$ locuteur d'obtenir une réponse précise, tout en gardant un minimum de politesse dans l'utilisation de la périphrase au conditionnel.

- sur les Jeux Olympiques d'hiver

- en sport

- J'aimerais savoir quel est le pays qui a participé à tous les Jeux Olympiques d'hiver: question nominale périphrastique $=$ reformulation claire et polie de la question du locuteur

- non c'est pas vraiment la géographie, c'est le sport - j'aimerais savoir quel pays a participé à tous les Jeux Olympiques d'hiver : réaiguillage et réitération de la même question = patience du locuteur qui souhaite faire avancer la compréhension du système

- non, je m'intéresse au sport, aux Jeux Olympiques: balisage du domaine, une nouvelle fois

- J'aimerais savoir quel pays a participé à la totalité des Jeux Olympiques d'hiver, il y en a un seul je sais mais je ne sais plus lequel c'est : balisage et réitération de la question à laquelle vient s'ajouter une formulation avec présentatif / impossibilité de dire * je ne sais plus quoi c'est ou *je ne sais plus c'est quoi mais possibilité de dire je ne sais plus ce que c'est. Il ne s'agit pas d'un procédé tonique interpellatif virulent mais d'un balisage appuyé sur un seul élément : volonté de nominaliser.

- sur rien, merci au revoir

Figure 4

Exemple de « cohorte » construit sur une question nominale avec réponse en ENLieu. Conscient de s'adresser à un interlocuteur « restreint » puisqu'il s'agit d'une machine, il n'interpelle son allocutaire que de manière mesurée. On peut penser qu'en présence d'un interlocuteur humain, il aurait pu formuler pour finir une question nominale tonique : Le pays qui a participé aux JO d'hiver, c'est lequel ? En outre, la présence d'une tonalité tonique reste indéniable dans cette "cohorte» ce qui montre que l'utilisateur du système a le sentiment que sa question est clairement posée. Il s'attend donc à une réponse valide correspondant pleinement aux critères mis en débat. Répondre à côté serait perçu comme une absence d'entendement chez l'interlocuteur ou comme une marque de mépris profond, et les questions toniques demeurent une forme d'interrogation interpellative. Bien sûr, ce type de corpora caractérisé par la fouille de données ne constitue pas réellement un dialogue autour d'une praxis. Cependant, la modalité interrogative qui le compose permet de cartographier plus précisément les variations morphosyntaxiques autour d'une même interrogation.

\section{Conclusions et perspectives}

Le concept de «réponse escomptée " établit un lien de fait entre morphosyntaxe, intentionnalité et interactivité. A partir d'indices structurels et formels, on se met en mesure d'appréhender le contenu intentionnel d'un message. En examinant les énoncés 
oraux, perçus et conçus au fil de leur énonciation, la GI s'intéresse avant tout au processus dynamique de l'interaction langagière. Les modèles ainsi mis au point cherchent à permettre sinon une meilleure compréhension des questions, du moins une capacité à prévoir une réaction non linéaire et, partant, une interaction plus efficace, susceptible de "réparer » par le dialogue les éventuels malentendus ou quiproquos parasites. Bien sûr, les quiproquos volontaires et conscients demeurent, ainsi que les altercations ou les incompréhensions entre interactants. Les questions toniques constituent un exemple révélateur de cette possibilité d'inférer à partir d'observables morphosyntaxiques des indices fiables de l'état des relations entre interlocuteurs. En effet, plus encore que l'objet même de la question, les protagonistes de l'échange cherchent à marquer leur territoire et à développer leurs relations intersubjectives, soit en montrant une politesse extrême, signe d'une véritable prise en considération, soit, à l'opposé, en attaquant la face positive d'un interlocuteur qu'ils tentent ainsi de placer en position de faiblesse, plus ou moins relative.

31 La GI, en travaillant à partir des indices morphosyntaxiques d'un corpus " actif ", offre une catégorisation non normative qui tente de mesurer l'utilisation réelle de la langue à travers l'intentionnalité du locuteur. Nous nous sommes ainsi efforcée de montrer le caractère interpellatif des questions toniques.

Deux types de perspectives s'offrent maintenant à nous concernant la validation des modèles de la GI. Linguistiquement, d'une part, il nous semble important de confronter le corpus RITEL à d'autres corpora (littéraires à travers un roman comme Poil de carotte de Jules Renard et sociologiques telles les enquêtes regroupées dans les corpora «Un sms pour la science ») afin de pallier les limites du corpus comme la non-réponse de l'interlocuteur machine. A ce propos, il est probable que les questions toniques interpellatives face à un interlocuteur humain, de fait "non restreint», soient plus nombreuses et parfois plus agressives. D'un point de vue informatique d'autre part, l'application de la GI dans un système de DOHM devrait permettre d'envisager à terme un système qui s'écarte enfin d'un schéma simple de Questions/Réponses pour aller vers un système en mesure de dialoguer mais aussi d'interpeller, ce qui suppose une capacité à manier politesse et agressivité.

\section{BIBLIOGRAPHIE}

Avanzi, M., Goldman, J.P., Lacheret-Dujour, A., Simon, A.C., Auchlin, A. (2007). « Méthodologie et Algorithmes pour la détection automatique des syllabes proéminentes dans les corpus de français parlé ", Cahiers of French Language Studies, 12/2, 2007.

Fourour N. (2004), Identification et catégorisation automatiques des entités nommées dans les textes français, thèse de doctorat, université de Nantes.

Kerbrat-Orecchioni C. (2005) nouveau tirage, Le discours en interaction, Paris : Armand Colin.

Luzzati, D. (2006), « Essai de description interactive : l'examen des questions quantificatrices », colloque « La quantification », PUC (à paraître). 
Rosset, S., Galibert O., Illouz G., Max A. (2005) «Interaction et recherche d'informations : le projet RITEL, de la langue française », TAL, Vol. $46, \mathrm{~N}^{\circ} 3,2007$.

Searle J. R., (1996) nouveau tirage, Les Actes de Langage, essai de philosophie du langage, Paris : Hermann.

\section{RÉSUMÉS}

Nous nous proposons d'étudier le cas de l'interrogation tonique (questions de type : tu vas où ? Ça coûte combien? Tu pars quand? Tu fais quoi ?) à travers le prisme de la "Grammaire Interactive " [Luzzati, 2006], qui présuppose que la morphosyntaxe d'une interrogation dépend directement dans l'esprit du locuteur d'une "réponse escomptée ", l'interlocuteur se réservant le droit de produire une réponse effective divergente. Cela revient à se fonder sur la morphosyntaxe des énoncés pour aborder le contenu intentionnel du langage en interaction, à la différence des approches essentiellement planificatrices fondées sur les théories des Actes de Langage [Searle, 1996]. En analysant à partir de cette théorie les variations morphosyntaxiques de l'interrogation temporelle, locative, quantificatrice à travers des corpora, on peut constater que les questions toniques sont certes rares mais qu'elles interviennent, à chaque fois, dans un contexte houleux, proche de la rupture. Outre le versant juridique de la signification du verbe interpeller, il faut donc souligner sa dimension énergique et participative. Lorsqu'une question tonique est posée, le locuteur interpelle directement son allocutaire. La "réponse escomptée » ne concerne pas tant l'objet de la question que le destinataire lui-même. Ce dernier reçoit alors, de manière insistante, une obligation de présence dans le discours, une injonction qui l'oblige moins à réponse qu'à se justifier et à expliciter son attitude.

In the present paper we propose to study the tonic interrogation in French thanks to an "Interactive Grammar". This grammar is an interpretative and dynamic grammar. It constitutes a direct link between question and answer and assumes that the morphosyntax of interrogation depends on an anticipated answer. We suggest an observation of question-answering corpora coming from the RITEL system showing the distribution of different morphosyntatic kinds of questions. By analyzing from this theoretical standpoint the morphosyntactic variations of interrogations on time, space, and quantity in text corpora, one can notice that tonic questions are certainly rare but that they appear, each time, in a contentious context, close to disruption. Besides the legal acception of the meaning of the verb interpellate, it is thus necessary to underline its energetic and participative dimension. So, we find an opposition between the tonic and periphrastic questions: the anticipated answer does not concern so much the object of the question as the interlocutor himself. He then perceives an obligation of presence in speech, compelling him less to answer than to justify himself and to clarify his attitude.

\section{INDEX}

Mots-clés : grammaire interactive, modèles dynamiques, question tonique, interpellation, morphosyntaxe

Keywords : interactive grammar, dynamic models, tonic question, morphosyntax 


\section{AUTEUR}

\section{CAROLE LAILLER}

Laboratoire d'informatique, Université du Maine (LIUM) 